\title{
Quantification and Classification of Corn and Sunflower Oils as Adulterants in Olive Oil Using Chemometrics and FTIR Spectra
}

\author{
Abdul Rohman'1,2 and Y. B. Che Man \\ ${ }^{1}$ Laboratory of Analytical Chemistry, Department of Pharmaceutical Chemistry, Faculty of Pharmacy, \\ Gadjah Mada University, Yogyakarta 55281, Indonesia \\ ${ }^{2}$ Research Center of Halal Products, Gadjah Mada University, Yogyakarta 55281, Indonesia \\ ${ }^{3}$ Laboratory of Analysis and Authentication, Halal Products Research Institute, Universiti Putra Malaysia, \\ Serdang 43400, Selangor, Malaysia \\ Correspondence should be addressed to Abdul Rohman, abdulkimfar@gmail.com
}

Received 10 October 2011; Accepted 16 November 2011

Academic Editor: Abdelhameed M. Othman

Copyright (c) 2012 A. Rohman and Y. B. Che Man. This is an open access article distributed under the Creative Commons Attribution License, which permits unrestricted use, distribution, and reproduction in any medium, provided the original work is properly cited.

\begin{abstract}
Commercially, extra virgin olive oil (EVOO) is subjected to be adulterated with low-price oils having similar color to EVOO. Fourier transform infrared (FTIR) spectroscopy combined with chemometrics has been successfully used for classification and quantification of corn (CO) and sunflower oils (SFOs) in EVOO sets. The combined frequency regions of 3027-3000, 1076-860, and 790-698 $\mathrm{cm}^{-1}$ were used for classification and quantification of CO in EVOO; meanwhile, SFO was analyzed using frequency regions of 3025-3000 and 1400-985 $\mathrm{cm}^{-1}$. Discriminant analysis can make classification of pure EVOO and EVOO adulterated with CO and SFO with no misclassification reported. The presence of CO in EVOO was determined with the aid of partial least square calibration using FTIR normal spectra. The calibration and validation errors obtained in CO's quantification are 0.404 and $1.13 \%$, respectively. Meanwhile, the first derivative FTIR spectra and PLS calibration model were preferred for quantification of SFO in EVOO with high coefficient of determination $\left(R^{2}\right)$ and low errors, either in calibration or in validation sample sets.
\end{abstract}

\section{Introduction}

Extra virgin olive oil (EVOO) is the highest classes of olive oil accounting for an approximately of $10 \%$ from olive oil production. Olive oil is among the most important oils used by humans. Olive has contributed to a great economic and social importance for the Mediterranean regions [1]. However, the olive oil is not strictly consumed by Mediterranean people. In the market, olive oil has high price; consequently, olive oil is subjected to be adulterated with other oils having similar color like corn and sunflower oils [2].

From economic reason, some unscrupulous market players may try to add lower-priced plant or nut oils to fresh EVOOs. This action is being unfair to the consumer because incorrect labeling can represent commercial deception [3]. In addition, the adulteration practice may also cause severe health and safety problems, especially to whom having allergy history [4]. Consequently, there is no doubt that the detection of adulteration needs to be addressed in order to ensure the quality of EVOO [5].

Chromatographic-based techniques such as high performance liquid chromatography $[6,7]$ and gas chromatography [8], especially in combination with mass spectrometer and expensive instruments like NMR spectroscopy [9], are the common analytical technique widely used for detection of EVOO adulteration. However, this technique involves excessive chemical reagents and solvents which are unsafe to human and environmental. For this reason, several efforts have been attempted to detect EVOO adulteration using greener techniques. Such methods are based on vibrational spectroscopic techniques of Raman [5] and infrared [10,11].

Vibrational spectroscopy can be taken into account as green analytical techniques owing to uselessness of chemical reagents and solvents.

Fourier transform infrared (FTIR) spectroscopy has emerged as powerful and alternative technique for wet and 
chromatographic methods because little sample preparation is needed, analysis is rapid, and the use of hazardous solvents is minimized. These analytical figures of merit result in time and cost savings and increase the number of analyzed samples [12]. With the aid of chemometric techniques, FTIR spectroscopy has been successfully used for classification and quantification of plant oil adulterants in EVOO. Such adulterants are sesame oil [13] and palm oil [14] quantified with partial least square (PLS) regression, sunflower, corn, soybean, and hazelnut oils using multiple linear regression and linear discriminant analysis [15], corn and sunflower oils using PLS-discriminant analysis [16]. The present study highlights the application of FTIR spectra combined with chemometrics techniques for classification and quantification of corn and sunflower oils after the FTIR spectra are subjected to several spectral treatments.

\section{Materials and Methods}

2.1. Materials. Extra virgin olive oil, corn, and sunflower oils were obtained from several supermarkets in Yogyakarta, Indonesia. In order to asses the purity of studied oils, fatty acid composition of oils was determined. The profiles of FA in these oils were compared with those specified in standard Codex [17]. Otherwise specified, all reagents and chemicals used during this study were bought from E. Merck (Darmstat, Germany). The standard of fatty acid methyl esters (C4-C24) was purchased from Sigma Aldrich (USA).

2.2. Fatty Acid Analysis. Fatty acid compositions of oil samples were determined with gas chromatography coupled with flame ionization detector (GC-FID). Fatty acid methyl esters (FAMEs) were prepared according to Cocks and van Rede [18]. An approximately $50 \mathrm{mg}$ of oil samples was dissolved in $1.0 \mathrm{~mL}$ hexane and added with $0.25 \mathrm{~mL}$ sodium methoxide $1 \mathrm{M}$. The mixture was vortexed and the upper layer containing FAME was transferred to $2 \mathrm{~mL}$ vial for a subsequent analysis using gas chromatograph (Agilent Technologies $6890 \mathrm{~N}$, Santa Clara, CA). The capillary column used was RESTEX 2330 (0.25 mm internal diameter, $30 \mathrm{~m}$ length and $0.2 \mu \mathrm{m}$ film thickness; Restek Corp, Bellefonte, $\mathrm{PA}$, USA) at a column pressure of $1.03 \times 10^{5} \mathrm{~Pa}$. The initial column temperature was $50^{\circ} \mathrm{C}$ (held for $2 \mathrm{~min}$ ), then increased to $180^{\circ}$ at a rate of $5^{\circ} \mathrm{C} / \mathrm{min}$, held for $2 \mathrm{~min}$ at $180^{\circ} \mathrm{C}$, then increased at a rate of $8^{\circ} \mathrm{C} / \mathrm{min}$ to $200^{\circ} \mathrm{C}$, and held for $5 \mathrm{~min}$ at $200^{\circ} \mathrm{C}$. Standard FAME from Sigma was used as authentic samples. The tentative peak identification was done by comparing the relative retention times of samples to those of FAMEs standard. Quantification of FAME was carried out based on internal normalization technique.

2.3. Classification. Classification of EVOO and EVOO adulterated with $\mathrm{CO}, \mathrm{SFO}$, and the mixture $\mathrm{CO}-\mathrm{SFO}$ was carried out using discriminant analysis (DA). In this stuy, a set of 20 EVOO samples and 20 EVOO samples adulterated with CO and SFO with concentration ranges of $2.0-50.0 \%(\mathrm{v} / \mathrm{v})$ was prepared. All samples were scanned with FTIR spectrometer.
2.4. Quantification. Quantification of CO and SFO was performed with the aid of multivariate calibrations, namely, principle component regression (PCR) and partial least square (PLS). For analysis of CO in EVOO, a set of 19 calibration samples and 19 validation samples was prepared in neat form comprising of $\mathrm{CO}$ in the concentration range of $1.0-50.0 \%(\mathrm{v} / \mathrm{v})$. Calibration and validation samples of SFO in EVOO were made similar to $\mathrm{CO}$ as above. All samples were measured with FTIR spectrometer.

2.5. FTIR Spectra Acquisition. All spectra of samples were scanned using FTIR spectrometer Nicolet from Thermo Nicolet Corp., Madison, WI, USA. This instrument was equipped with DTGS detector and $\mathrm{KBr} /$ Germanium beam splitter. The operating system used was the OMNIC software (Version 7.0, Thermo Nicolet, Madison, WI, USA). The sampling compartment was Smart Attenuated Total Reflectance kit (Smart ARK, Thermo Electron Corp.) with dimension of $10 \times 60 \mathrm{~mm}$. The Smart ARK is an advanced multi-bounce horizontal attenuated reflectance accessory, producing 12 internal reflections with a penetration depth (infrared beam) of $2.0 \mu \mathrm{m}$. The accessory was composed of zink selenide ( $\mathrm{ZnSe}$ ) crystal with an aperture angle of $45^{\circ}$ and refractive index of 2.4 at $1000 \mathrm{~cm}^{-1}$. FTIR spectra were acquired at region of $4000-650 \mathrm{~cm}^{-1}$ at coaddition 32 interferograms and resolution of $4 \mathrm{~cm}^{-1}$ with strong apodization. These spectra were subtracted against the background of air spectrum. After every scan, a background of new reference air spectrum was taken. The ATR plate was carefully cleaned using soft tissue soaked in hexane and acetone for removing any residues coming from previous samples. The ATR cleanliness was monitored by collecting a background spectrum and compared to the previous one. These spectra were recorded as absorbance values at each data point in triplicate.

2.6. Chemometrics. Discriminant analysis and multivariate calibrations employing partial least square (PLS) and principle component regression (PCR) were performed by TQ Analyst Software (Thermo electron Corporation) included in FTIR spectrometer. The difference between actual and calculated values of corn and sunflower oils in calibration model was calculated as root mean square error of calibration (RMSEC). The predictive ability of PLS was assessed by computing root mean square error of prediction (RMSEP) and $R^{2}$ values.

\section{Results and Discussion}

3.1. Fatty Acid Composition. It has been explained by some authors that fatty acid composition has been known to affect the exact position and intensity of peaks due to the proportion of saturated and unsaturated fatty acids $[19,20]$. SFO and CO exhibited a maximum absorbance at $3009 \mathrm{~cm}^{-1}$, while EVOO has maximum peak absorbance at $3006 \mathrm{~cm}^{-1}$. The shift of spectral band was attributed from differences in the proportion of oleic acid acyl groups and linoleic and 
TABLE 1: FA composition of olive, corn, and sunflower oils.

\begin{tabular}{lccc}
\hline & & The FA composition of studied oils & SFO \\
\hline $\mathrm{C} 14: 0$ & EVOO & CO & $0.03 \pm 0.01$ \\
$\mathrm{C} 16: 0$ & $0.02 \pm 0.00$ & $0.06 \pm 0.02$ & $6.81 \pm 0.06$ \\
$\mathrm{C} 16: 1$ & $10.48 \pm 0.12$ & $12.70 \pm 0.45$ & $0.03 \pm 0.01$ \\
$\mathrm{C} 18: 0$ & $0.66 \pm 0.01$ & $0.10 \pm 0.01$ & $3.99 \pm 0.15$ \\
$\mathrm{C} 18: 1$ & $3.20 \pm 0.02$ & $2.01 \pm 0.08$ & $36.86 \pm 1.92$ \\
$\mathrm{C} 18: 2$ & $71.50 \pm 1.15$ & $27.48 \pm 0.26$ & $44.08 \pm 0.33$ \\
$\mathrm{C} 20: 0$ & $10.65 \pm 0.29$ & $53.24 \pm 0.92$ & $0.65 \pm 0.00$ \\
$\mathrm{C} 18: 3$ & $0.05 \pm 0.01$ & $0.09 \pm 0.00$ & $3.68 \pm 0.15$ \\
$\mathrm{C} 20: 1$ & $0.65 \pm 0.01$ & $0.73 \pm 0.02$ & $0.40 \pm 0.02$ \\
$\mathrm{C} 22: 0$ & $0.46 \pm 0.01$ & $0.43 \pm 0.01$ & $0.57 \pm 0.01^{\mathrm{g}}$ \\
\hline
\end{tabular}

TABLE 2: The performance of multivariate calibration (MC) and spectral treatments for analysis of sunflower oil in EVOO.

\begin{tabular}{|c|c|c|c|c|c|c|c|c|}
\hline \multirow[b]{2}{*}{$\mathrm{MC}$} & \multirow[b]{2}{*}{ Spectra } & \multirow[b]{2}{*}{ Factor } & \multicolumn{2}{|c|}{ Equation } & \multicolumn{2}{|c|}{$R^{2}$} & \multirow{2}{*}{$\begin{array}{c}\mathrm{RM} \\
\mathrm{SEC} \\
(\% \mathrm{v} / \mathrm{v})\end{array}$} & \multirow{2}{*}{$\begin{array}{c}\text { RM } \\
\text { SEP } \\
(\% \mathrm{v} / \mathrm{v}) \\
\end{array}$} \\
\hline & & & Calibration & Prediction & Calibration & Prediction & & \\
\hline \multirow{3}{*}{ PLS } & Normal & (8) & $y=1.000 x-0.001$ & $y=1.097 x-0.378$ & 1.000 & 0.997 & 0.005 & 2.34 \\
\hline & $1 s t$ der & (8) & $y=1.000 x-0.002$ & $y=1.015 x+0.139$ & 1.000 & 0.987 & 0.034 & 2.02 \\
\hline & 2nd der & (7) & $y=0.999 x+0.017$ & $y=0.512 x+9.505$ & 0.999 & 0.580 & 0.425 & 10.3 \\
\hline \multirow{3}{*}{ PCR } & Normal & (9) & $y=0.999 x+0.020$ & $y=1.125 x-0.447$ & 0.999 & 0.975 & 0.426 & 4.07 \\
\hline & 1st der & (9) & $y=0.997 x+0.067$ & $y=0.958 x+1.085$ & 0.997 & 0.985 & 0.829 & 1.98 \\
\hline & 2nd der & (9) & $y=0.901 x+1.976$ & $y=0.083 x+11.612$ & 0.901 & 0.052 & 4.44 & 17.2 \\
\hline
\end{tabular}

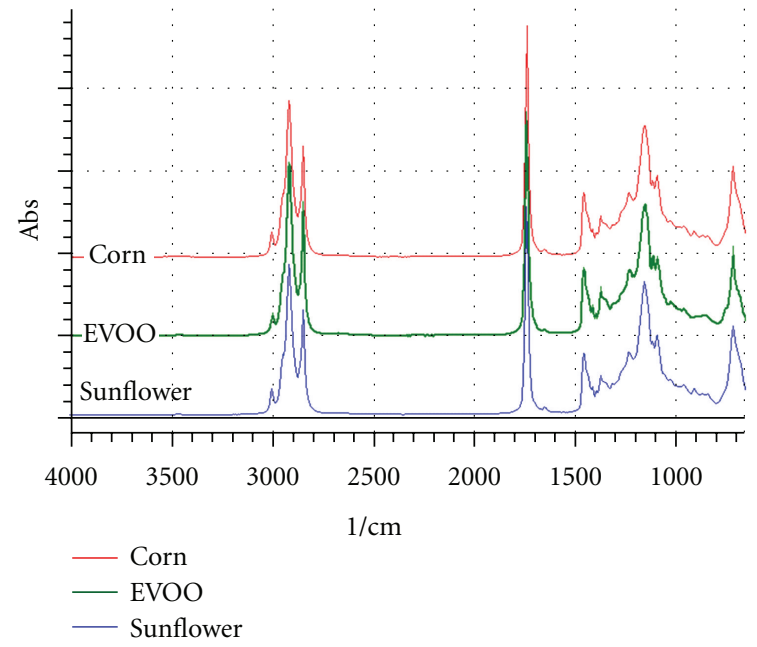

FIGURE 1: FTIR spectra of extra virgin olive oil, corn oil, and sunflower oil scanned at wavenumbers of 4000-650 1/cm.

linolenic acyl groups [20]. Fatty acid composition of SFO, $\mathrm{CO}$, and EVOO was shown in Table 1.

3.2. FTIR Spectral Analysis. The characteristics of midinfrared spectra for extra virgin olive oil (EVOO), corn oil (CO), and sunflower oil (SFO) are shown in Figure 1. These spectra look very similar and showed a typical characteristic of absorption peaks for common triglyceride, main component composed edible fats and oils. Band at $3007 \mathrm{~cm}^{-1}$ is attributed from the stretching vibration of $=\mathrm{C}-\mathrm{H}$. Strong band absorptions were observed in the region of 3000$2800 \mathrm{~cm}^{-1}$ caused by corresponding to $\mathrm{C}-\mathrm{H}$ stretching vibrations. The strectching vibrations of methylene $\left(-\mathrm{CH}_{2}-\right)$ and methyl $\left(-\mathrm{CH}_{3}\right)$ groups can be seen at frequencies of 2922 and $2853 \mathrm{~cm}^{-1}$, respectively. Methylene and methyl groups are also observed at $1465 \mathrm{~cm}^{-1}$ and $1377 \mathrm{~cm}^{-1}$ due to their bending vibrations. The large peak around $1740 \mathrm{~cm}^{-1}$ is due to $\mathrm{C}=\mathrm{O}$ double bond stretching vibration. Deformation and bending of $\mathrm{C}-\mathrm{H}$ and stretching vibration of $\mathrm{C}-\mathrm{O}$ result in peaks in the $1500-650 \mathrm{~cm}^{-1}$ region [21].

The differences among them were clearly small and occurred only in limited regions of the spectra, especially in peak intensities at fingerprint regions $\left(1500-650 \mathrm{~cm}^{-1}\right)$ and at 3007 or $3009 \mathrm{~cm}^{-1}$. The selection of frequency regions used for analysis was automatically suggested by software; however, analyst should evaluate this region by observing the differences between EVOO and adulterants (CO and SFO).

3.3. Classification Analysis. Classification of EVOO and EVOO adulterated with SFO and CO was performed with discriminant analysis (DA) using frequency regions of 3027$3000,1076-860$, and $790-698 \mathrm{~cm}^{-1}(\mathrm{CO})$ and at frequency regions of $3025-3000$ and $1400-985 \mathrm{~cm}^{-1}$ for SFO. These frequencies offer good model for classification. In this study, EVOO was adulterated with SFO and CO individually. 


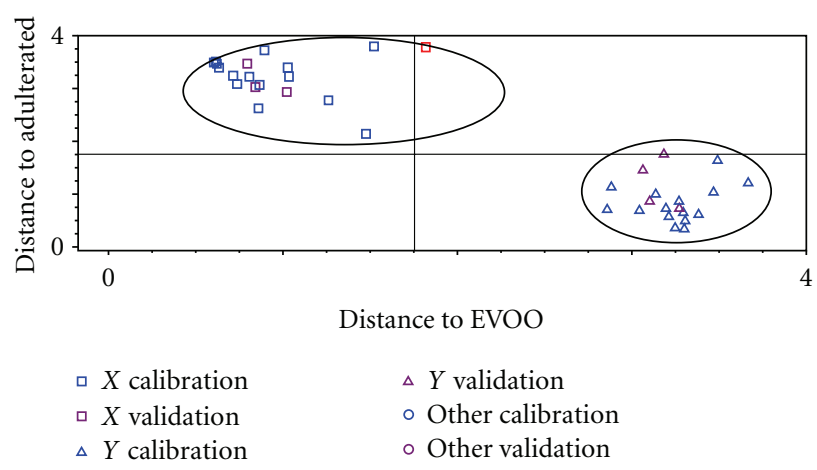

(a)

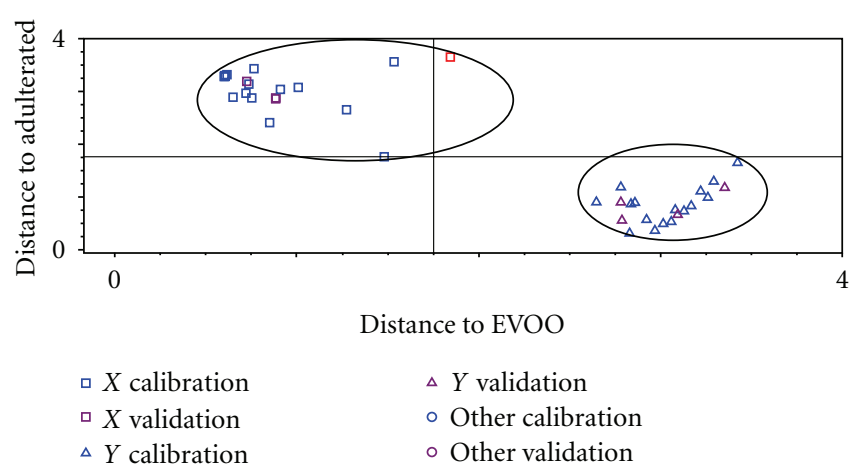

(b)

FIgUre 2: The Coomans plot for classification of EVOO and EVOO adulterated with corn oil (a) and with sunflower oil (b).

TABLE 3: The performance of multivariate calibration (MC) and spectral treatments for analysis of corn oil in EVOO.

\begin{tabular}{ccccccccc}
\hline MC & Spectra & Factor & Calibration & Prediction & Calibration & $\begin{array}{c}R^{2} \\
\text { Prediction }\end{array}$ & $\begin{array}{c}\text { RM } \\
\text { SEC } \\
(\% \mathrm{v} / \mathrm{v})\end{array}$ & $\begin{array}{c}\text { RM } \\
\text { SEP } \\
(\% \mathrm{v} / \mathrm{v})\end{array}$ \\
\hline \multirow{2}{*}{ PLS } & Normal & $(3)$ & $y=0.999 x+0.013$ & $y=0.969 x+1.363$ & 0.999 & 0.997 & 0.404 & 1.13 \\
& 1 st der & $(7)$ & $y=1.000 x-0.001$ & $y=0.936 x+1.519$ & 1.000 & 0.977 & 0.019 & 2.34 \\
& 2nd der & $(8)$ & $y=0.999 x-0.001$ & $y=0.567 x+5.439$ & 1.000 & 0.534 & 0.083 & 10.5 \\
\hline \multirow{2}{*}{ PCR } & Normal & $(10)$ & $y=0.999 x+0.014$ & $y=0.970 x+1.136$ & 0.999 & 0.997 & 0.394 & 1.17 \\
& 1 1st der & $(10)$ & $y=0.999 x+0.012$ & $y=0.931 x+1.462$ & 0.999 & 0.977 & 0.356 & 2.33 \\
& 2nd der & $(10)$ & $y=0.995 x+0.095$ & $y=0.567 x+5.338$ & 0.995 & 0.497 & 1.02 & 11.0 \\
\hline
\end{tabular}

Figures 2(a) and 2(b) showed the Coomans plot calculated based on the Mahalanobis distance of EVOO adulterated with SFO and CO. The Mahalanobis distance of EVOO mixed with adulterants to EVOO was described in $x$-axis; meanwhile, the distance of EVOO to EVOO added with adulterants was shown in $y$-axis.

The modeled DA can successfully make the classification between EVOO and EVOO adulterated with $\mathrm{CO}$ and SFO with no misclassification reported. This means that DA can classify both classes with accuracy level of 100\%. Sometimes, the misclassification can occur for some reasons, namely, (i) the close similarity in terms of chemical composition between adulterants and EVOO and (ii) the frequency regions used are not appropriate.

3.4. Quantification. Quantification of $\mathrm{CO}$ and SFO was carried out with the aid of multivariate calibrations. Two calibration models, namely, partial least square (PLS) and principle component regression (PCR) were used to evaluate the goodness of fit for the relationship between actual value $(x$-axis) and FTIR predicted value ( $y$-axis) of CO and SFO in EVOO. Table 3 compiled the performance of PLS and PCR for quantification of CO in EVOO. Based on Table 3, the first derivative spectra offers the highest coefficient of determination (1.000) and the lowest errors in calibration model expressed as root mean square error in calibration or RMSEC of $0.019 \% \mathrm{v} / \mathrm{v}$ ), however, this model shows the high error in prediction model expressed with root mean square error of prediction (RMSEP) of $2.34 \% \mathrm{v} / \mathrm{v}$. In addition, the number of factors used is to high ( 8 factors).This means that over-fitting occurs for such model.

Overfitting the regression model is one of the potential disadvantages when using PLS regression [22]. It means that the model generates an optimistic model on the set of data used for calibration (low value of RMSEC), but the model would not perform well on other datasets with similar material, usually used in validation dataset (high value of RMSEP). For this reason, the presence of CO in EVOO was better quantified with PLS using FTIR normal spectra for the reason that low RMSEC value $(0.404 \% \mathrm{v} / \mathrm{v})$ was followed with low error in RMSEP $(1.13 \% \mathrm{v} / \mathrm{v})$. Figure 3 exhibited the closed relationship between these two parameters either in calibration or validation sample sets.

Furthermore, the presence of SFO in EVOO was better quantified using PLS with first derivative spectra (Table 2). Among others, PLS with first derivative spectra gives the reasonable $R^{2}$ either in calibration or in validation and offers the acceptable errors in calibration and validation. Using this model, 8 factors are needed to obtained RMSEC value of 0.034 and RMSEP value of $2.02 \% \mathrm{v} / \mathrm{v}$. the scatter plot for the relationship between actual value ( $x$-axis) and FTIR predicted value ( $y$-axis) of SFO in EVOO was revealed in Figure 4. Based on this result, it can be deduced that FTIR spectroscopy with the appropriate selection of calibration model and spectral treatment can facilitate the detection and quantification of $\mathrm{CO}$ and $\mathrm{SFO}$ as adulterants in EVOO. 


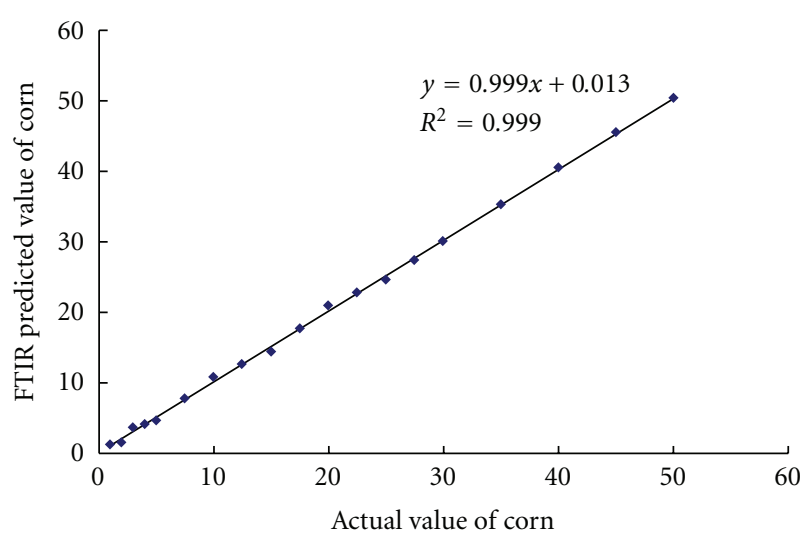

(a)

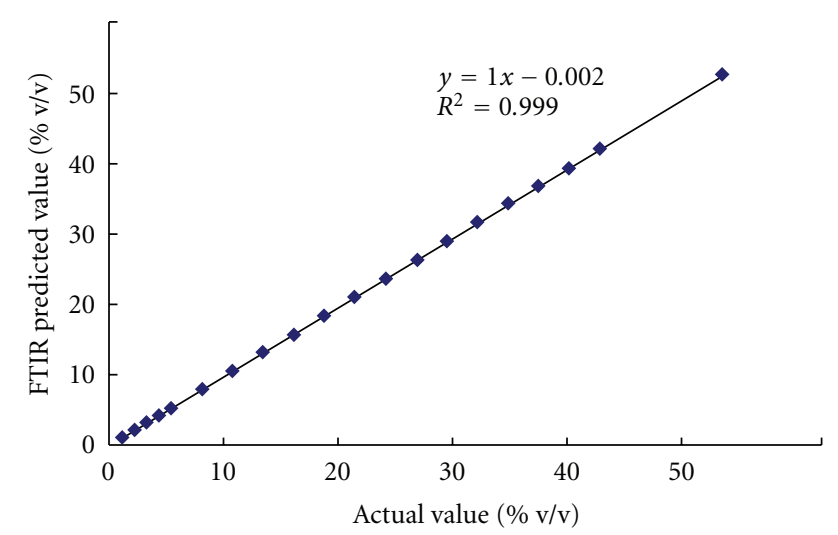

(b)

FIGURe 3: PLS model for the relationship between actual value and FTIR predicted value of corn oil in EVOO using FTIR normal spectra at $790-698,1076-860$, and $3027-3000 \mathrm{~cm}^{-1}$; (a) calibration; (b) validation.

The developed method is fast and not using the toxic and hazardous solvents and reagents.

\section{Conclusions}

FTIR spectroscopy in combination with discriminant analysis (DA) offers an easy way for classification of EVOO and EVOO adulterated with $\mathrm{CO}$ and SFO. DA can accurately classify both classes without any sample misclassification reported. Quantification of CO and SFO as adulterants in EVOO using PLS calibration gives a good calibration and validation model with acceptable errors. The developed method is rapid, free from sample preparation, and not requiring the use of chemicals and reagents; therefore, FTIR technique can be considered as green analytical tools for classification and quantification of EVOO's adulterants.

\section{Acknowledgment}

This study was supported by Directorate of Higher Education, National Education Ministry by giving Abdul Rohman a financial scholarship during his Ph.D. study.

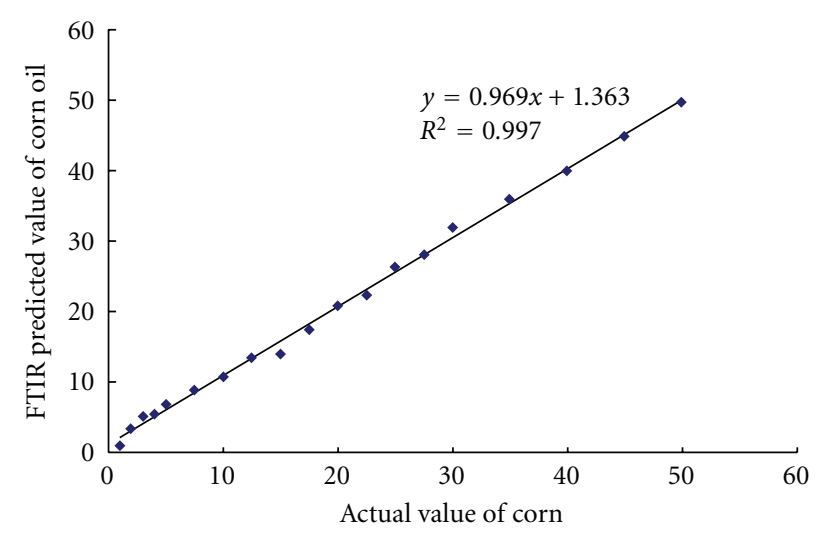

(a)

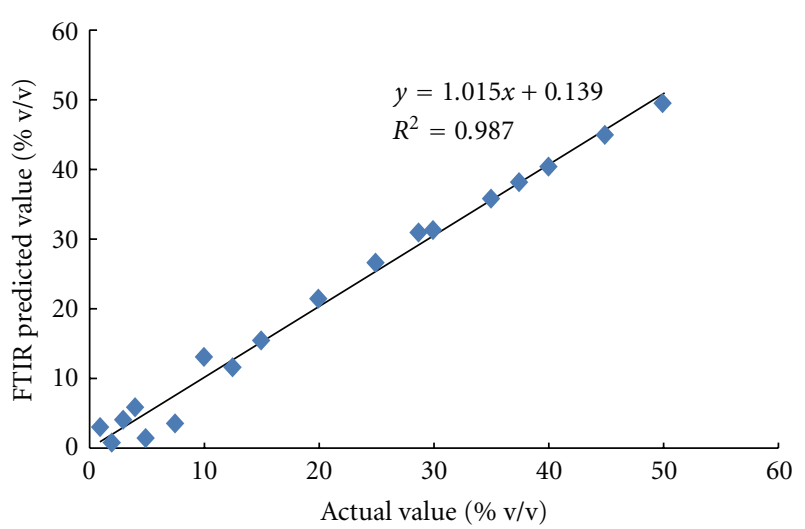

(b)

FIGURE 4: PLS model for relationship between actual value and FTIR predicted value of sunflower oil using FTIR 1st derivative spectra at 3025-3000 and 1400-985 $\mathrm{cm}^{-1}$; (a) calibration; (b) validation.

\section{References}

[1] J. E. Pardo, M. A. Cuesta, A. Alvarruiz, J. D. Granell, and M. Álvarez-Ortí, "Evaluation of potential and real qualities of virgin olive oil from the designation of origin (DO) " Aceite Montes de Alcaraz" (Albacete, Spain)," Food Chemistry, vol. 124, no. 4, pp. 1684-1690, 2011.

[2] G. Downey, P. McIntyre, and A. N. Davies, "Detecting and quantifying sunflower oil adulteration in extra virgin olive oils from the Eastern Mediterranean by visible and near-infrared spectroscopy," Journal of Agricultural and Food Chemistry, vol. 50, no. 20, pp. 5520-5525, 2002.

[3] S. Azadmard-Damirchi, "Review of the use of phytosterols as a detection tool for adulteration of olive oil with hazelnut oil," Food Additives and Contaminants: Part A, vol. 27, no. 1, pp. 1-10, 2010.

[4] M. Arlorio, J. D. Coisson, M. Bordiga et al., "Olive oil adulterated with hazelnut oils: simulation to identify possible risks to allergic consumers," Food Additives and Contaminants: Part A, vol. 27, no. 1, pp. 11-18, 2010.

[5] E. C. López-Díez, G. Bianchi, and R. Goodacre, "Rapid quantitative assessment of the adulteration of virgin olive oils with hazelnut oils using Raman spectroscopy and chemometrics," Journal of Agricultural and Food Chemistry, vol. 51, no. 21, pp. 6145-6150, 2003. 
[6] F. P. Capote, J. R. Jiménez, and M. D. L. de Castro, "Sequential (step-by-step) detection, identification and quantitation of extra virgin olive oil adulteration by chemometric treatment of chromatographic profiles," Analytical and Bioanalytical Chemistry, vol. 388, no. 8, pp. 1859-1865, 2007.

[7] M. Fasciotti and A. D. P. Netto, "Optimization and application of methods of triacylglycerol evaluation for characterization of olive oil adulteration by soybean oil with HPLC-APCI-MSMS," Talanta, vol. 81, no. 3, pp. 1116-1125, 2010.

[8] C. Ruiz-Samblás, A. González-Casado, L. Cuadros-Rodríguez, and F. P. R. García, "Application of selected ion monitoring to the analysis of triacylglycerols in olive oil by high temperaturegas chromatography/mass spectrometry," Talanta, vol. 82, no. 1, pp. 255-260, 2010.

[9] A. Agiomyrgianaki, P. V. Petrakis, and P. Dais, "Detection of refined olive oil adulteration with refined hazelnut oil by employing NMR spectroscopy and multivariate statistical analysis," Talanta, vol. 80, no. 5, pp. 2165-2171, 2010.

[10] P. de la Mata, A. Dominguez-Vidal, J. M. Bosque-Sendra, A. Ruiz-Medina, L. Cuadros-Rodríguez, and M. J. Ayora-Cañada, "Olive oil assessment in edible oil blends by means of ATRFTIR and chemometrics," Food Control, vol. 23, no. 2, pp. 449 455, 2012.

[11] M. de Luca, W. Terouzi, G. Ioele et al., "Derivative FTIR spectroscopy for cluster analysis and classification of morocco olive oils," Food Chemistry, vol. 124, no. 3, pp. 1113-1118, 2011.

[12] L. E. Rodriguez-Saona and M. E. Allendorf, "Use of FTIR for rapid authentication and detection of adulteration of food," Annual Review Food Science and Technology, vol. 2, pp. 17.1$17.17,2011$.

[13] A. Rohman and Y. B. Che Man, "Authentication of extra virgin olive oil from sesame oil using FTIR spectroscopy and gas chromatography," International Journal of Food Properties. In press.

[14] A. Rohman and Y. B. Che Man, "Fourier transform infrared (FTIR) spectroscopy for analysis of extra virgin olive oil adulterated with palm oil," Food Research International, vol. 43, no. 3, pp. 886-892, 2010.

[15] M. J. Lerma-García, G. Ramis-Ramos, J. M. Herrero-Martínez, and E. F. Simó-Alfonso, "Authentication of extra virgin olive oils by Fourier-transform infrared spectroscopy," Food Chemistry, vol. 118, no. 1, pp. 78-83, 2010.

[16] S. M. Obeidat, M. S. Khanfar, and W. M. Obeidat, "Classification of edible oils and uncovering adulteration of virgin olive oil using FTIR with the aid of chemometrics," Australian Journal of Basic and Applied Sciences, vol. 3, no. 3, pp. 20482053, 2009.

[17] Codex Allimentareus Commision. 2003.

[18] L. V. Cocks and C. van Rede, Laboratory Handbook for Oil and Fats Analysts, Academic Press, London, UK, 1966.

[19] M. Safar, D. Bertrand, P. Robert, M. F. Devaux, and C. Genot, "Characterization of edible oils, butters and margarines by Fourier transform infrared spectroscopy with attenuated total reflectance," Journal of the American Oil Chemists' Society, vol. 71, no. 4, pp. 371-377, 1994.

[20] N. Vlachos, Y. Skopelitis, M. Psaroudaki, V. Konstantinidou, A. Chatzilazarou, and E. Tegou, "Applications of Fourier transform-infrared spectroscopy to edible oils," Analytica Chimica Acta, vol. 573-574, pp. 459-465, 2006.

[21] G. Gurdeniz, F. Tokatli, and B. Ozen, "Differentiation of mixtures of monovarietal olive oils by mid-infrared spectroscopy and chemometrics," European Journal of Lipid Science and Technology, vol. 109, no. 12, pp. 1194-1202, 2007.
[22] J. N. Miller and J. C. Miller, Statistics and Chemometrics for Analytical Chemistry, Pearson Education Limited, Edinburgh Gate Harlow, England, 5th edition, 2005. 


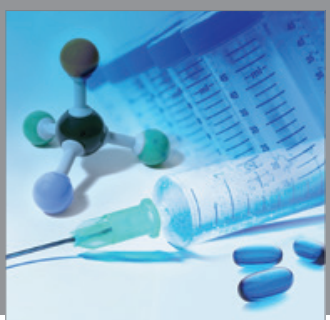

International Journal of

Medicinal Chemistry

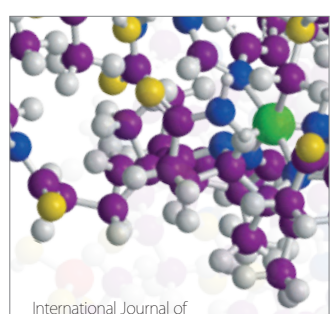

Carbohydrate Chemistry

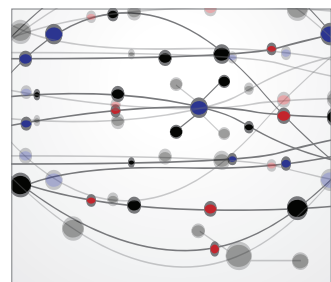

The Scientific World Journal
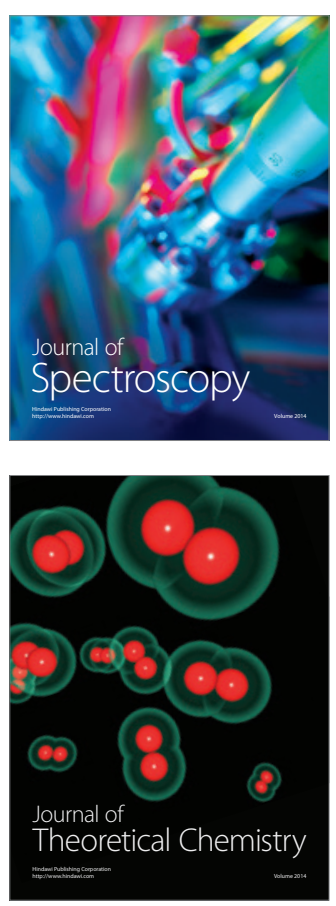
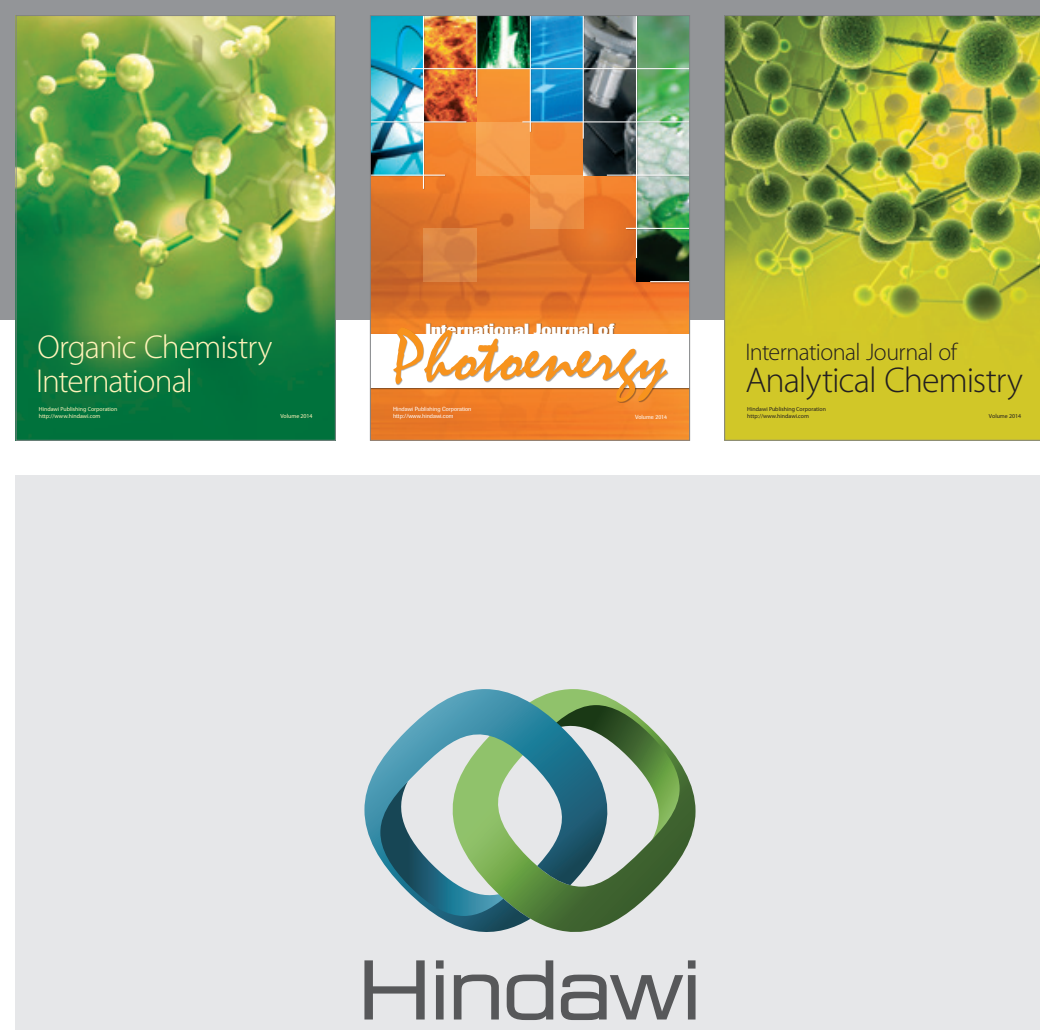

Submit your manuscripts at

http://www.hindawi.com
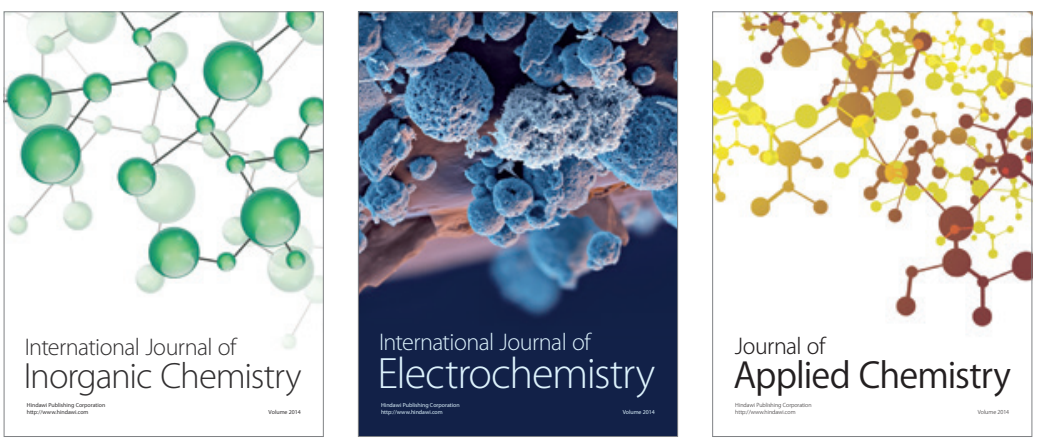

Journal of

Applied Chemistry
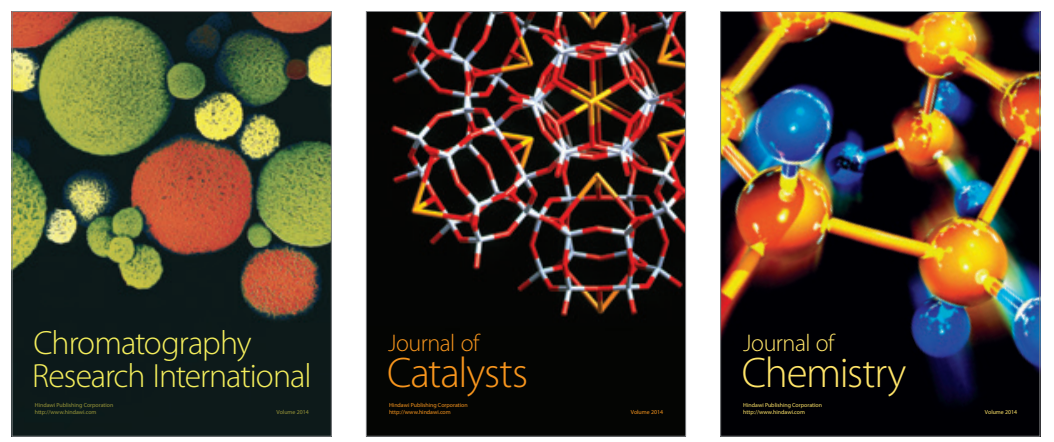
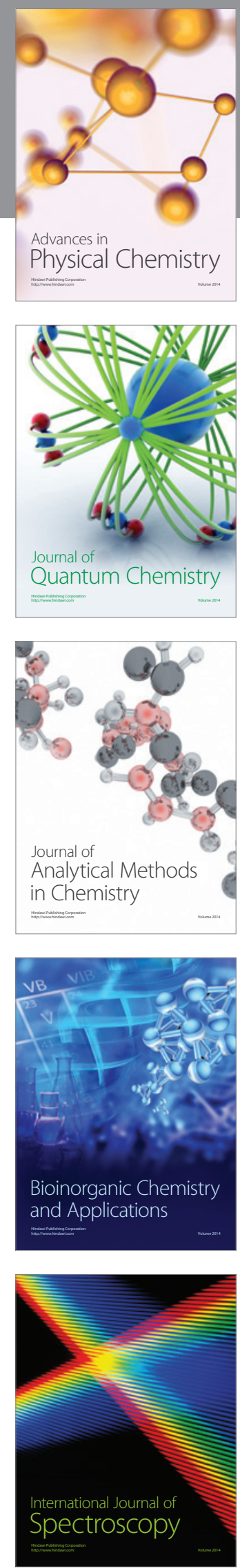